\title{
economia

\section{Identificando a persistência e os determinantes dos desvios inflacionários no Brasil}

\author{
André Abdala ${ }^{1}$ \\ Ricardo Ramalhete Moreira ${ }^{2}$
}

\begin{abstract}
Resumo: Este artigo identificou o componente de persistência e os determinantes dos desvios inflacionários no Brasil, entre jan/2003 e dez/2014. Realizaram-se estimações por GMM, e de respostas generalizadas ao impulso através de modelos Vetoriais Autoregressivos Bayesianos (BVAR). Verificou-se que: i) as estimações por GMM indicam elevado grau de inércia de tais desvios, apesar de uma atenuação nas estimações BVAR; ii) variação cambial e de preços de commodities não pode ser creditada como principal fonte dos desvios inflacionários; iii) a credibilidade desempenha papel importante nesses desvios.
\end{abstract}

Palavras-chave: desvios inflacionários; persistência; inércia; Brasil.

\section{Identifying the persistence and the determinants of inflation deviations in Brazil}

\begin{abstract}
This work identified the persistent component and the determinants of inflation deviations in Brazil, from Jan/2003 to Dec/2014. We performed GMM estimates, and an analysis of generalized impulse-response by Bayesian Vector Autoregressive (BVAR) models. We found that: i) The GMM estimates suggested a high degree of inertia of such deviations, despite it was mitigated with the BVAR models; ii) exchange rate and commodity prices changes cannot be regarded as the main sources for such deviations; iii) credibility plays an important role in these deviations.
\end{abstract}

Keywords: inflation deviations; persistence; inertia; Brazil.

Classificação JEL: E310; E37; E58.

\footnotetext{
${ }^{1}$ Mestre em Economia pela Universidade Federal do Espírito Santo (UFES). E-mail: andre_abdala@hotmail.com

${ }^{2}$ Doutor em Economia pela Universidade Federal do Rio de Janeiro (UFRJ). Professor do Programa de PósGraduação em Economia da Universidade Federal do Espírito Santo (UFES). E-mail: ramalhete.s@gmail.com
} 


\section{Introdução}

Este trabalho tem como principal objetivo investigar os determinantes dos desvios inflacionários no Brasil ao longo desse período de análise. Embora haja trabalhos empíricos que estudaram os determinantes da taxa de inflação brasileira (Curva de Phillips), tais como Cysne (1985), Veloso et al. (2013) e Ponzoni \& Zilli (2015), não há ainda amplas evidências empíricas quando se deseja conhecer os fatores que afetam não o nível ou a variação da taxa de inflação, mas sim o nível dos desvios da inflação em relação à meta inflacionária. Embora sutil, a diferença é crucial porque a eficiência de um regime de inflation targeting está em eliminar os desvios inflacionários no longo prazo. Como será argumentado, isso implica no ideal de que a série temporal de desvios inflacionários seja, do ponto de vista estatístico, um processo estocástico com média zero e variância constante, ideal este que pode não ser verificado na prática.

Os resultados indicam que há elevado grau de inércia de tais desvios e que a variação cambial e de preços de commodities não pode ser creditada como principal fonte dos desvios inflacionários, além de evidenciar que a credibilidade desempenha papel importante nesses desvios.

O trabalho está estruturado da seguinte maneira: na próxima seção são apresentados os elementos pertinentes em nível teórico e uma breve revisão da literatura empírica subjacente; já a seção subsequente apresenta a base de dados deste trabalho, a metodologia empírica e a análise dos resultados obtidos.

\section{Desvios inflacionários no regime de metas de inflação: aspectos teóricos}

Barro e Gordon (1983) avaliam que existem dois tipos de regimes de políticas monetárias: a política pela regra e a política discricionária. Na primeira, a política tem como principal objetivo a estabilidade da inflação no longo prazo. Na segunda, a autoridade monetária tem a flexibilidade de atuar de modo oposto às expectativas dos agentes econômicos para obter ganhos de produto ou emprego em curto prazo. Contudo, a violação da regra tem como consequência a formação de expectativas de inflação com base em valores observados e não com base no objetivo anunciado pelas autoridades, de modo que há uma perda de bem-estar e maior custo inflacionário.

Nesse sentido, Clarida et al. (1999) demonstram que a política ideal é a política sob o compromisso, pela melhor aderência da trajetória do produto à consecução da meta inflacionária. Já Svensson (1997) pondera que quanto maior o peso da estabilização do produto enquanto objetivo de política, maior a dificuldade para a convergência da inflação corrente ou futura à meta de inflação, de modo que 
a política ótima deve desconsiderar metas adicionais, como o produto e emprego, no longo prazo.

Após a adoção de uma meta de inflação oficial no Brasil (ou a partir de 1999), observou-se uma tendência a desvios inflacionários persistentes no período como um todo, ou seja, a desvios da taxa de inflação ao consumidor em relação à meta inflacionária estabelecida como guia de política monetária, em especial quando observamos o ocorrido em anos mais recentes. Contudo, a literatura sobre inflation targeting mostra que um regime de metas para inflação eficiente está associado à eliminação de tais desvios no longo prazo (Svensson, 1997; Ball, 1999, 1999b; Clarida et al. 1999; Woodford, 2001). Isso significa que embora possa haver desvios inflacionários transitórios, como resposta à necessidade de acomodação ou flexibilidade diante de choques, em longo prazo esses desvios não podem persistir como um evento esperado, a fim de que o regime de metas para inflação seja considerado eficiente e conquiste credibilidade para a política monetária.

No Gráfico 1 é possível observar o comportamento dos desvios da inflação efetiva (acumulada nos últimos 12 meses) e das expectativas de inflação (acumulada para 12 meses à frente), ambas em relação à meta de inflação, no período de janeiro de 2003 a dezembro de 2014. Embora as séries mostrem valores positivos e negativos ao longo do tempo, e mesmo iguais a zero em alguns meses, a partir de meados de 2010 os desvios iniciaram um processo de persistência positiva. De fato, a média do desvio da inflação efetiva no período como um todo foi de 1,43 (ou seja, em média, a inflação efetiva ficou 1,43 ponto percentual acima da meta de inflação no período jan/2003-dez/2014) e a média do desvio das expectativas foi de 0,43 . 
Gráfico 1 - Desvios da inflação observada (linha tracejada) e desvios das expectativas de inflação (linha cheia) frente à meta, com base no IPCA (janeiro de 2003 a dezembro de 2014)

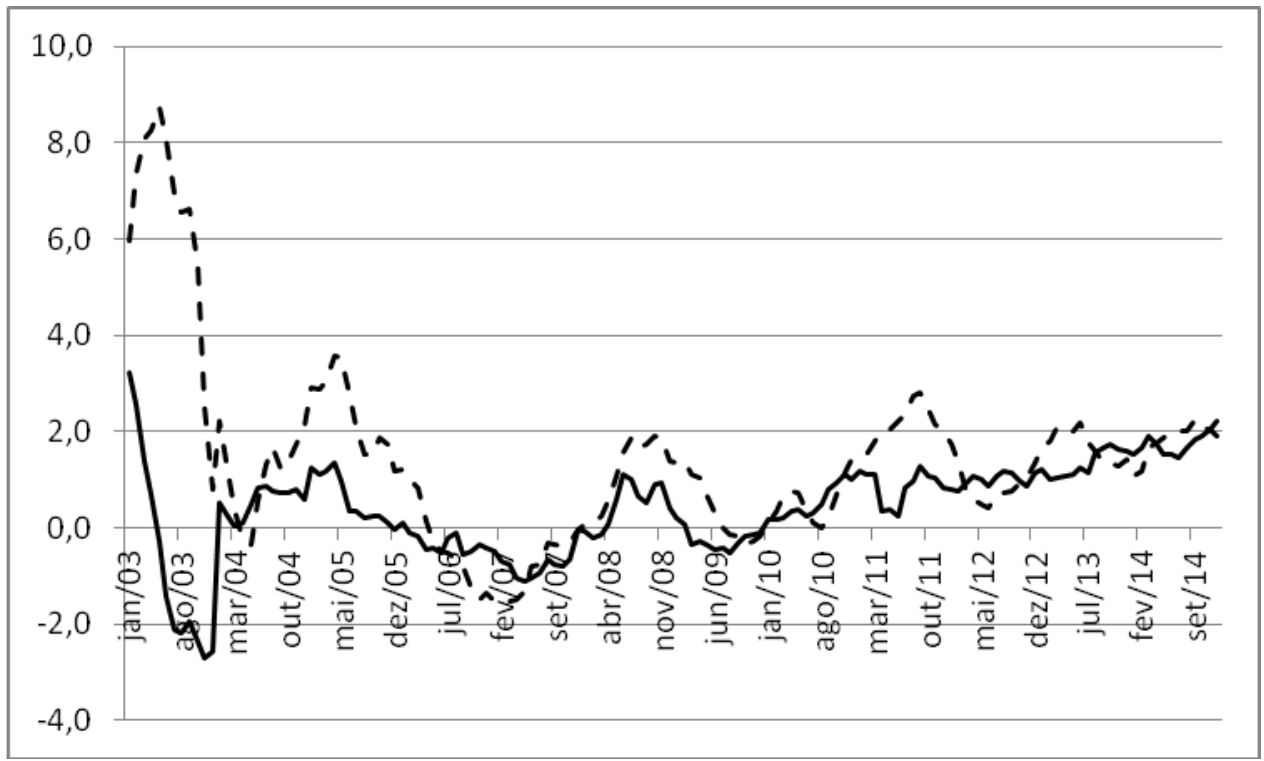

Fonte: Elaboração própria com dados do BCB.

Sob o regime de inflation targeting o objetivo do Banco Central no longo prazo é convergir a inflação observada ( $\Pi$ ) - entendida como a diferença entre o nível de preços atual e o nível de preços do período anterior $\left(P_{t}-P_{t-1}\right)$ - na meta de inflação $\left(\Pi^{T}\right)$, esta última fixada pela autoridade. Seja o comportamento da inflação expresso da seguinte maneira:

$$
\Pi \sim\left(\Pi^{T}+d_{\Pi}^{2}\right)
$$

Em que $\delta^{2} \Pi$ é a variância da inflação. Portanto, pode-se entender (1) como a condição de eficiência do regime de metas para inflação. Assim, a inflação observada converge para a meta de inflação e os desvios não apresentam tendência de mudança. Nessa situação, as expectativas de inflação E[ П] são ancoradas na meta de inflação, tal que:

$$
\mathrm{E}[\Pi]=\Pi^{T}
$$

Pode-se definir o desvio inflacionário então como a diferença entre a inflação observada e a meta de inflação. Sendo assim: 


$$
\Pi=\prod-\Pi^{T}
$$

Em um regime de metas de inflação cuja condição de eficiência é satisfeita (1), tem-se que $p=0$. No entanto, caso essa condição não seja satisfeita tem-se que $p \neq$ $\mathrm{o}$, ou $\mathrm{p}=\mathrm{k}$, sendo $\mathrm{k}$ uma constante positiva ou negativa, o que caracteriza a persistência dos desvios. Portanto, quando o regime de metas de inflação é ineficiente, tem-se que:

$$
\Pi \sim\left(\Pi^{T}+\mathrm{k}, \mathrm{d}_{\Pi}^{2}\right)
$$

Logo, se a condição de eficiência não é satisfeita, a inflação converge para um valor acima ou abaixo da meta de inflação, cujo desvio persistente é dado por k. A implicação disso é uma espécie de punição em termos de perda de credibilidade da meta de inflação, que deixa de ancorar as expectativas. Estas passam a se comportar tal que:

$$
\mathrm{E}[\Pi]=\Pi^{T}+\mathrm{k}
$$

Dessa maneira, a fim de que o regime de metas para inflação consiga satisfazer a condição de eficiência e eliminar a persistência dos desvios, é fundamental identificar e testar para os possíveis determinantes desses desvios. Uma equação que explique o comportamento de $\mathrm{k}$ no tempo é necessária, como forma de explicar as variáveis de controle dessa perda social.

Em geral, pode-se apresentar os seguintes potenciais determinantes dos desvios inflacionários, com base na literatura moderna: i) inércia inflacionária; ii) fatores ligados à atividade econômica, tais como taxa de desemprego e gap do produto; iii) fatores expectacionais, tais como as expectativas inflacionárias; iv) dinâmica cambial (efeito pass-through); v) custos ou choques de oferta, a exemplo da dinâmica dos preços de insumos e commodities; os fatores i a v estão presentes em trabalhos que partem da Curva de Phillips novo-keynesiana, tais como em Svensson (1997), Clarida, et al. (1999), e Ponzoni e Zilli (2015); vi) fatores fiscais, a exemplo da dívida pública na hipótese da Teoria Fiscal do Nível de Preços (Woodford, 2001).

Dado o histórico de hiperinflação no Brasil anterior ao Plano Real, foram publicados alguns trabalhos nas últimas décadas que analisaram o fenômeno da inércia inflacionária e de suas implicações em termos de política econômica. De Holanda Barbosa e Mcnelis (1989) atestaram que as tentativas do governo brasileiro no sentido de indexar salários e taxas de câmbio à inflação passada ao longo dos anos pré-Plano Real teve como resultado a perda de eficácia das políticas monetária e fiscal e fez com que a dinâmica inflacionária se tornasse mais sensível 
a choques de oferta. Novaes (1993) também testou a hipótese de inflação inercial para a economia brasileira no período pré-Plano Real por meio de técnicas uni e multivariadas, obtendo resultados mostrando a existência de inércia, porém não em níveis tão elevados como esperado. Essa inércia abaixo do que alguns modelos teóricos supunham também foi estimada por Durevall (1998), que estudou o período de 1968-1985, enquanto Cati et al. (1999) avaliaram o baixo grau de persistência dos choques das políticas econômicas, entre 1974 e 1993, tendo em vista que esses choques tornam os testes de raiz unitária tendentes à estacionariedade, considerando, em especial, que esse viés é maior em planos de curta duração.

Por sua vez, Gottschalk (2003) estimou mudança de regimes na inércia inflacionária brasileira e identificou uma quebra estrutural a partir do Plano Real, tratando a inflação como um processo tendência-estacionário. Já Reisen et al. (2003) aplicaram uma abordagem de memória longa ao invés de raiz unitária e, por meio da estimação de diferenciação fracional via modelo Arfima para a taxa de inflação, concluíram que não haveria processo inercial na inflação brasileira, em contraste com os resultados anteriores. Por fim, mais recentemente e testando para o período pós-regime de metas inflacionárias, Cerisola e Gelos (2009) encontraram resultados mostrando maior ancoragem do processo de formação de expectativas de inflação, com papel importante para a instância fiscal nesse processo, além de terem sugerido a não existência de inércia substancial na dinâmica inflacionária a partir de 1999.

Já Figueiredo e Marques (2009, 2011) observam uma queda da memória inflacionária, a partir da implementação do Plano Real. Então, Figueiredo e Marques (2011), por meio do uso de markov e da integração fracionária na média e na variância, adotam o modelo MS-ARFIMA, ao avaliar dois tipos de regimes: um regime de alta inflação e outro regime de baixa inflação. O primeiro regime é marcado pelas fases de industrialização e da década de 1980 até o início do decênio seguinte. E no segundo regime está delimitado pelo início do período de industrialização, década de 1970 e após o Plano Real. Assim, observam alta persistência no regime de baixa inflação, o que é consistente teoricamente com a adoção do Plano Real, visto que a maior mudança entre os dois regimes se encontra quando há redução no grau de indexação da economia.

Em corroboração, Gomes da Silva e Vieira (2013) encontram persistência inflacionária controlada na maior parte das regiões metropolitanas nacionais, ao longo de agosto de 1999 a dezembro de 2011. E, igualmente, Gomes da Silva e Leme (2011) verificam baixo grau de persistência inflacionária brasileira entre meados de 1999 e 2011, assim, como Campêlo e Cribari-Neto (2003), sendo que, nesse caso, observam um período mais longo (1944-200o), ante a aplicação de testes de raiz 
unitária para identificar a estacionariedade e, por efeito, a presença de uma inércia em longo prazo, ao aceitar a hipótese nula.

Todos esses trabalhos aplicados à economia brasileira deram atenção especial à inércia da taxa de inflação. No entanto, há ainda uma lacuna na literatura relacionada no que diz respeito à inércia dos desvios inflacionários, ou seja, à inércia dos desvios da taxa de inflação face à meta inflacionária, tal como Machado e Portugal (2014), ao observarem que, entre 1995 e 2011, a persistência ou o desvio das expectativas de inflação, em relação à meta da política, é um fator relevante para a existência da persistência inflacionária no Brasil. Como argumentado, sob um regime de metas de inflação a análise de eficiência do regime pauta-se na dinâmica dos desvios, muito mais do que na dinâmica do nível da taxa inflacionária.

\section{Implementação empírica}

\subsection{Dados}

As estimações econométricas realizadas neste trabalho fizeram uso de séries temporais em frequência mensal que compreendem o período de janeiro de $2003 \mathrm{a}$ dezembro de 2014. A seguir é feita a descrição de cada uma delas.

Desvio da inflação (p): Essa é a variável endógena ou dependente das regressões estimadas. Representa a diferença entre a inflação acumulada em doze meses e a meta de inflação definida pelo $\mathrm{BCB}\left(\mathrm{p}_{t}=\prod_{t}-\Pi^{T}\right)$, com base no Índice Nacional de Preços ao Consumidor Amplo (IPCA/IBGE). Fonte: BCB;

Taxa de câmbio (e): Taxa no fim do período (R\$/US\$ venda). Fonte: Ipeadata;

Desvio de expectativa de inflação $(E(p))$ : Representa a diferença entre a expectativa de inflação acumulada em doze meses à frente e a meta de inflação definida pelo $\mathrm{BCB}\left(\mathrm{E}(\mathrm{p})_{t}=\mathrm{E}(\Pi)_{t}-\Pi^{T}\right)$. Fonte: $\mathrm{BCB}$;

Dívida líquida interna do setor público (\% PIB) (dlsp): participação percentual da Dívida Líquida Interna do Setor público no PIB. Fonte: BCB;

Índice de atividade econômica do $B C B$ - IBC-BR (ibc): Esse índice é uma representação do PIB em frequência mensal, mensurado pelo $B C B$ e serve às decisões de política monetária. Fonte: $\mathrm{BCB}$; 
Índice de commodities do $B C B$ - IC-BR (ic): índice de commodities calculado pelo BCB. Faz a conversão dos preços de uma cesta de commodities para R\$. Fonte: $\mathrm{BCB}$;

Indice de credibilidade Cecchetti e Krause (2002) ( cred $\left._{C K}\right)$ : O índice varia entre o e 1, e foi calculado pelas seguintes condições:

$$
I C^{C K}=\left\{\begin{array}{c}
1, \text { se } E(\Pi) \leq \Pi^{T} \\
1-\frac{1}{20 \%-\Pi^{T}}\left[E(\Pi)-\Pi^{T}\right], \text { se } \Pi^{T}<E(\Pi)<20 \% \\
0, \text { se } E(\Pi) \geq 20 \%
\end{array}\right.
$$

Índice de credibilidade de Mendonça (2007) ( $\left.\operatorname{cred}_{M}\right)$ : O índice varia entre o e 1, e foi calculado pelas seguintes condições:

$$
I C^{M E}=\left\{\begin{array}{c}
1, \text { se } E(\Pi)=\Pi^{T} \\
1-\frac{1}{\Pi_{\max }^{T}-\Pi^{T}}\left[E(\Pi)-\Pi^{T}\right] \text {, se } \Pi_{\min }^{T}<E(\Pi)<\Pi_{\max }^{T} \\
0 \text {, se } E(\Pi) \geq \Pi_{\max }^{T} \text { ou } E(\Pi) \leq \Pi_{\min }^{T}
\end{array}\right.
$$

Taxa de juros Selic (i): Taxa efetiva de juros Selic anualizada. Fonte: Ipeadata;

Índice de commodities geral do FMI (icfmi): usado para substituir o IC-Br na análise de robustez. Fonte: FMI;

Taxa de desocupação (\%) (u): Usada para substituir o IBC na análise de robustez. Fonte: IBGE.

Tabela 1: Estatísticas descritivas

\begin{tabular}{ccccccccccccc}
\hline & $\mathbf{E}$ & $E(\pi)$ & $\pi$ & dlsp & ibc & Ic & cred $_{\mathrm{CK}}$ & cred $_{\mathbf{M}}$ & i & icfmi & $\mathbf{u}$ \\
\hline Média & 2,1862 & 0,4306 & 1,4399 & 46,2425 & 127,2313 & 112,4759 & 0,9565 & 0,5932 & 13,2413 & 140,0766 & 8,1243 \\
Mediana & 2,1337 & 0,5100 & 1,3400 & 46,3750 & 127,5300 & 105,0200 & 0,9659 & 0,6180 & 11,7500 & 140,8331 & 7,9500 \\
Máximo & 3,5632 & 3,2200 & 8,7400 & 51,8400 & 148,7000 & 151,9000 & 1,0026 & 0,9840 & 26,5000 & 219,8995 & 13,1000 \\
Mínimo & 1,5563 & $-2,7000$ & $-1,5400$ & 41,4500 & 98,9500 & 86,6700 & 0,7200 & 0,0000 & 7,2500 & 60,6892 & 4,3000 \\
$\begin{array}{c}\text { Desvio } \\
\text { padrão }\end{array}$ & 0,4512 & 0,9940 & 1,9431 & 2,6474 & 15,3901 & 17,4680 & 0,0465 & 0,2771 & 4,6431 & 43,8977 & 2,4888 \\
Assimetria & 0,8220 & $-0,6049$ & 1,7180 & 0,1838 & $-0,2383$ & 0,6513 & $-1,5260$ & $-0,5246$ & 1,1414 & $-0,2077$ & 0,2964 \\
Curtose & 3,0950 & 3,9894 & 6,9223 & 2,0591 & 1,7141 & 2,1147 & 7,3614 & 2,3355 & 3,8920 & 1,8067 & 1,9642 \\
\hline
\end{tabular}

Fonte: Elaboração própria. 


\subsection{Estratégia metodológica}

Foram implementados os seguintes testes de raiz unitária: Augmented Dickey-Fuller (ADF), Generalized Least Squares Detrending (DF-GLS) e PhillipsPerron (PP), com objetivo de identificar a ordem de integração das séries temporais. A Tabela 2 apresenta os resultados obtidos.

Tabela 2: Testes de raiz unitária - ADF, PP e DF-GLS

\begin{tabular}{c|ccc|cccc}
\hline & ADF $^{1}$ & PP $^{2}$ & DF-GLS & ADF $^{4}$ & PP $^{5}$ & DF-GLS & \\
\hline Séries & \multicolumn{3}{|c|}{ Teste em nível } & & Teste em $1^{\circ}$ diferença & $\mathrm{I}(\mathrm{n})$ \\
\hline $\mathrm{e}$ & $-1,5430$ & $-1,5430$ & $-0,3322$ & $-11,5099^{* * *}$ & $-11,5117^{* * *}-10,5019^{* * *}$ & $\mathrm{I}(1)$ \\
$E(\pi)$ & $-4,7839^{* * *}$ & $-5,1116^{* * *}$ & $-1,3027$ & - & - & - & $\mathrm{I}(\mathrm{o})$ \\
$\pi$ & $-3,8676^{* * *}$ & $-2,5206^{* *}$ & $-2,0622$ & - & - & - & $\mathrm{I}(\mathrm{o})$ \\
dlsp & $-2,0358$ & $-1,3935$ & $-1,6794^{*}$ & $-5,2612^{* * *}$ & $-8,7058^{* * *}$ & $-5,0116$ & $\mathrm{I}(1)$ \\
ibc & $-2,6050$ & $-1,9988$ & $-2,6761$ & $-9,7616^{* * *}$ & $-9,8430^{* * *}$ & $-5,7878^{* * *}$ & $\mathrm{I}(1)$ \\
ic & $-3,3232$ & $-2,5880$ & $-2,5737$ & $-9,2215^{* * *}$ & $-9,152^{* * *}$ & $-6,5507^{* * *}$ & $\mathrm{I}(1)$ \\
cred & $-7,6064^{* * *}$ & $-6,8035^{* * *}$ & $-0,6813$ & - & - & - & $\mathrm{I}(\mathrm{o})$ \\
cred & $-3,8118^{* *}$ & $-3,8751^{* *}$ & $-1,9506$ & - & - & - & $\mathrm{I}(\mathrm{o})$ \\
$\mathrm{i}$ & $-5,2425^{* * *}$ & $-1,9441^{* *}$ & $-2,1071$ & - & - & - & $\mathrm{I}(\mathrm{o})$ \\
icfmi & $-2,3267$ & $-2,0779$ & $-2,4802$ & $-6,6781^{* * *}$ & $-6,7340^{* * *}$ & $-6,6189^{* * *}$ & $\mathrm{I}(1)$ \\
$\mathrm{u}$ & $-3,8684^{* *}$ & $-4,5385^{* * *}$ & $-3,6892^{* * *}$ & - & - & - & $\mathrm{I}(\mathrm{o})$ \\
\hline
\end{tabular}

Obs.: $\left(^{*}\right)$ nível de significância de $10 \%,\left({ }^{* *}\right) 5 \%$ e $\left.{ }^{* * *}\right) 1 \%$. O nível de significância para a constante e a tendência pode variar entre 1 e $10 \%$. (1) O desvio da inflação é significante sem a constante e a tendência; i é significante sem a constante e a tendência. (3) dlsp significante apenas com a constante. (4) dlsp é significante sem a constante e a tendência; ibc é significante apenas com a constante; o ic é significante sem a constante e a tendência; o icfmi é significante sem a constante e a tendência. (5) dlsp é significante sem a constante e a tendência; ibc é significante apenas com a constante; icfmi é significante sem a constante e a tendência. (6) A diferença do cred $\mathrm{CK}_{\mathrm{C}}$ é maior do que 2. Fonte: Elaboração própria.

Com base no conjunto de testes de raiz unitária, somente as variáveis câmbio, dlsp, ibc, ic e icfmi são integradas de primeira ordem, I(1), enquanto as demais são estacionárias em nível, I(o). Desse modo, estas últimas foram usadas em nível nas regressões estimadas, ao passo que as séries I(1) foram implementadas em suas respectivas primeiras diferenças.

Conforme Mendonça e Tostes (2015) e Montes e Tiberto (2015), o GMM, por sua vez, permite eliminar problemas de correlação serial, heterocedasticidade e não-linearidade comumente presentes em estimações com séries temporais por 
MQO. Entretanto, Cragg (1983) observa que o método de momentos, ou seja, o método que utiliza variáveis auxiliares ou instrumentais, aplica-se de acordo com o nível de heterocedasticidade, com maior eficiência em grandes amostras. Inclusive Wooldridge (2001) observa que o GMM busca um estimador ótimo que, além de eliminar os problemas de heterocedasticidade, garante a exogeneidade entre as variáveis explicativas e o termo de erro, de modo a garantir a menor variância nos estimadores do método de momentos do que nos estimadores de MQO.

Por fim, as estimações das regressões foram implementadas para cada índice de credibilidade adotado no trabalho, ou seja, para cada um desses índices foi estimado um conjunto de especificações em cada um dos métodos mencionados. Após isso, ainda foram implementadas novas estimações por GMM, nos quais as variáveis ibc e ic foram substituídas, respectivamente, por u e icfmi.

Sabe-se que nas relações entre desvios inflacionários e os canais de transmissão da política monetária existem em geral defasagens temporais expressivas, e que os efeitos de tais defasagens nem sempre são capturados por estimações de regressões por GMM. Nesse sentido, os resultados obtidos com esses métodos foram comparados aos obtidos por meio de uma análise de respostas generalizadas aos impulsos (Lutkenpohl, 1991), que por sua vez foram extraídas a partir de duas especificações de modelo Vetorial Autoregressivo Bayesiano (BVAR). Como apontado em Koop et. al. (1996) e Pesaran e Shin (1998), as funções impulsoresposta generalizadas dispensam a ordenação de Cholesky ou de endogeneidade/exogeneidade entre as séries do modelo, pois a respostas estimadas são robustas face à mudança dessa ordenação.

Quanto aos modelos BVAR, Litterman (1986) argumenta que em tais modelos os parâmetros são considerados como variáveis aleatórias e com probabilidades previamente definidas (priors). É então importante adotar um método para a definição dessa informação a priori a ser imposta sobre a média e a variância dos parâmetros. Koop e Korobilis (2009) sugerem o método de Litterman/Minnesota, embora haja outros métodos possíveis tais como Sims-Zha, Plosser-Rebelo, entre outros. Para Chauvet e Potter (2012), esse procedimento via BVAR resulta na substituição da matriz de variância-covariância por um processo de estimação baseado no filtro de Kalman.

\subsection{Resultados por GMM}

Em (6), define-se o modelo econométrico padrão, que servirá de base para todas as especificações estimadas por GMM:

$\pi_{\mathrm{t}}=\beta+\alpha_{1} \mathrm{e}_{\mathrm{t}-1}+\alpha_{2} \mathrm{E}(\pi)_{\mathrm{t}-1}+\alpha_{3} \pi_{\mathrm{t}-1}+\alpha_{4} \mathrm{dlsp}_{\mathrm{t}-1}+\alpha_{5} \mathrm{yjt}_{\mathrm{jt}-1}+\alpha_{6} \mathrm{comm}_{\mathrm{jt}-1}+\alpha_{7} \mathrm{i}_{\mathrm{t}-1}+\alpha_{8} \mathrm{cred}_{\mathrm{jt}-1}+\theta_{\mathrm{t}}$ 
Definimos então o desvio inflacionário $\left(\pi_{\mathrm{t}}\right)$ em $\mathrm{t}$ como dependente de uma constante $\beta$, dos componentes defasados para a taxa de câmbio $\mathrm{e}_{\mathrm{t}-1}$, as expectativas para desvios inflacionários futuros $\mathrm{E}(\pi)_{\mathrm{t}-1}$, a persistência ou inércia dos desvios $\pi_{\mathrm{t}-1}$, a dívida interna líquida do setor público face ao PIB dlsp $\mathrm{P}_{\mathrm{t}-1}$, para o indicador $\mathrm{j}$ de atividade econômica (sendo $\mathrm{y}_{j}=\mathrm{ibc}, \mathrm{u}$ ), para o indicador $\mathrm{j}$ de preços de commodities (sendo comm $\mathrm{j}_{\mathrm{j}} \mathrm{ic}$, icfmi), para a taxa Selic $\mathrm{i}_{\mathrm{t}-1}$ e para o indicador $\mathrm{j}$ de credibilidade ( $\operatorname{sendo} \mathrm{cred}_{\mathrm{j}}=\mathrm{cred}_{\mathrm{CK}}, \mathrm{cred}_{\mathrm{M}}$ ), sendo $\theta_{\mathrm{t}}$ representativo dos resíduos das estimações.

Para cada índice de credibilidade adotado no trabalho foi estimado um conjunto de especificações (o8 especificações). Na Tabela 3, apresentam-se inicialmente as estimações para cred $_{\mathrm{CK}}$, enquanto na Tabela 4 são mostrados os resultados para cred $_{\mathrm{M}}$.

Tabela 3: Desvio da inflação ( $\pi$ ): GMM com variável de cred ${ }_{\mathrm{CK}}$

\begin{tabular}{|c|c|c|c|c|c|c|c|c|}
\hline \multirow{2}{*}{$\begin{array}{c}\text { Variáveis } \\
\text { explanatórias }\end{array}$} & \multicolumn{8}{|c|}{ GMM } \\
\hline & Eq.1 & Eq.2 & Eq.3 & Eq.4 & Eq. 5 & Eq.6 & Eq.7 & Eq.8 \\
\hline \multirow[t]{3}{*}{ Constante } & $-6,2909$ & $-8,2183$ & $-9,1643$ & $-8,0566$ & $-7,1329$ & 0,0532 & $-6,0433$ & $-8,0566$ \\
\hline & $(10,0661)$ & $(9,4894)$ & $(6,4794)$ & $(5,9579)$ & $(5,1832)$ & $(0,0502)$ & $(5,7512)$ & $(5,9579)$ \\
\hline & {$[-0,6250]$} & {$[-0,8660]$} & {$[-1,4144]$} & {$[-1,3523]$} & {$[-1,3762]$} & {$[1,0595]$} & {$[-1,0508]$} & {$[-1,3523]$} \\
\hline \multirow[t]{3}{*}{$d(e)_{t-1}$} & 0,4206 & $-0,1022$ & $-0,9802$ & $-0,6766$ & $-0,8555$ & $-0,4766$ & $-0,8750$ & $-o, 6766$ \\
\hline & $(2,3066)$ & $(2,1315)$ & $(1,3204)$ & $(1,2250)$ & $(1,1540)$ & $(0,9566)$ & $(1,1898)$ & $(1,2250)$ \\
\hline & {$[0,1823]$} & {$[-0,0479]$} & {$[-0,7423]$} & {$[-0,5524]$} & {$[-0,7413]$} & {$[-0,4982]$} & {$[-0,7354]$} & {$[-0,5524]$} \\
\hline \multirow[t]{3}{*}{$E(\pi)_{t-1}$} & 0,3232 & 0,4233 & $0,4621^{*}$ & $0,4481^{* *}$ & $0,4145^{* *}$ & $0,1157^{* * *}$ & $0,3555^{*}$ & $0,4481^{* *}$ \\
\hline & $(0,3949)$ & $(0,3646)$ & $(0,2393)$ & $(0,2203)$ & $(0,1922)$ & $(0,0422)$ & $(0,2048)$ & $(0,2203)$ \\
\hline & {$[0,8184]$} & {$[1,1612]$} & {$[1,9313]$} & {$[2,0342]$} & {$[2,1565]$} & {$[2,7427]$} & {$[1,7357]$} & {$[2,0342]$} \\
\hline \multirow[t]{3}{*}{$\pi_{t-1}$} & $0,9734^{* * *}$ & $0,9475^{* * *}$ & $0,9389^{* * *}$ & $0,8923^{* * *}$ & $0,8868^{* * *}$ & $0,8799^{* * *}$ & $0,8898^{* * *}$ & $0,8923^{* * *}$ \\
\hline & $(0,1276)$ & $(0,1171)$ & $(0,0710)$ & $(0,0400)$ & $(0,0371)$ & $(0,0293)$ & $(0,0374)$ & $(0,0400)$ \\
\hline & {$[7,6303]$} & {$[8,0885]$} & {$[13,2174]$} & {$[22,3249]$} & {$[23,9279]$} & {$[30,0777]$} & {$[23,7675]$} & {$[22,3249]$} \\
\hline \multirow[t]{3}{*}{$\mathrm{d}(\mathrm{dlsp})_{\mathrm{t}-1}$} & $-0,6463$ & 0,1486 & & & & & $-0,0818$ & \\
\hline & $(1,4278)$ & $(0,8946)$ & & & & & $(0,4436)$ & \\
\hline & {$[-0,4527]$} & {$[0,1661]$} & & & & & {$[-0,1844]$} & \\
\hline \multirow[t]{3}{*}{$\mathrm{d}(\mathrm{ibc})_{\mathrm{t}-1}$} & 0,1239 & & & & & & & \\
\hline & $(0,1742)$ & & & & & & & \\
\hline & {$[0,7116]$} & & & & & & & \\
\hline $\mathrm{d}(\mathrm{ic})_{\mathrm{t}-1}$ & $-0,0004$ & 0,0346 & 0,0127 & o,0079 & & & & o,oo79 \\
\hline
\end{tabular}


(continuação)

\begin{tabular}{|c|c|c|c|c|c|c|c|c|}
\hline & $\begin{array}{c}(0,0719) \\
{[-0,0057]}\end{array}$ & $\begin{array}{l}(0,0508) \\
{[0,6802]} \\
\end{array}$ & $\begin{array}{l}(0,0270) \\
{[0,4699]}\end{array}$ & $\begin{array}{l}(0,0262) \\
{[0,3009]}\end{array}$ & & & & $\begin{array}{l}(0,0262) \\
{[0,3009]}\end{array}$ \\
\hline $\mathrm{i}_{\mathrm{t}-1}$ & $\begin{array}{l}-0,0054 \\
(0,0364) \\
{[-0,1474]}\end{array}$ & $\begin{array}{l}-0,0098 \\
(0,0345) \\
{[-0,2836]}\end{array}$ & $\begin{array}{c}-0,0136 \\
(0,0225) \\
{[-0,6046]}\end{array}$ & & & & & \\
\hline cred $_{\mathrm{CK}} \mathrm{t-1}$ & $\begin{array}{c}6,4615 \\
(10,3845) \\
{[0,6222]}\end{array}$ & $\begin{array}{l}8,5283 \\
(9,7572) \\
{[0,8741]}\end{array}$ & $\begin{array}{c}9,5615 \\
(6,6497) \\
{[1,4379]}\end{array}$ & $\begin{array}{l}8,2939 \\
(6,0838) \\
{[1,3633]}\end{array}$ & $\begin{array}{l}7,3599 \\
(5,3053) \\
{[1,3873]}\end{array}$ & & $\begin{array}{c}6,2570 \\
(5,8962) \\
{[1,0612]}\end{array}$ & $\begin{array}{c}8,2939 \\
(6,0838) \\
{[1,3633]}\end{array}$ \\
\hline $\mathrm{R}^{2}$ ajustado & 0,8830 & 0,9168 & 0,9155 & o,9176 & 0,9181 & 0,9295 & 0,9195 & 0,9176 \\
\hline Estatística J & 4,5559 & 5,3094 & 9,2365 & 9,1212 & 9,4465 & 9,9623 & 8,6982 & 9,1212 \\
\hline Prob. (J) & 0,3360 & 0,3793 & 0,1607 & 0,2441 & 0,306o & 0,3535 & 0,2751 & 0,2441 \\
\hline
\end{tabular}

Obs.: ( ) para desvio padrão e [ ] para estatística t. $\left(^{*}\right)$ nível de significância de $10 \%,\left({ }^{* *}\right) 5 \%$ e $\left({ }^{* * *}\right) 1 \%$. As variáveis instrumentais são $E(p)_{t-2}, d(e)_{t-2}, \mathrm{i}_{\mathrm{t}-2}, \mathrm{~d}(\mathrm{ic})_{\mathrm{t}-2}, E(p)_{t-3}, d(e)_{t-3}, \mathrm{i}_{\mathrm{t}-3}, \mathrm{~d}(\mathrm{ic})_{\mathrm{t}-3}, E(p)_{t-4}, d(e)_{t-4}$, $\mathrm{i}_{\mathrm{t}-4}, \mathrm{~d}(\mathrm{ic})_{\mathrm{t}-4}$. Fonte: Elaboração própria.

Tabela 4: Desvio da inflação ( $\pi$ ): GMM com variável de cred $_{M}$

\begin{tabular}{|c|c|c|c|c|c|c|c|c|}
\hline \multirow{2}{*}{$\begin{array}{c}\text { Variáveis } \\
\text { explanatórias }\end{array}$} & \multicolumn{8}{|c|}{ GMM } \\
\hline & Eq.1 & Eq.2 & Eq.3 & Eq.4 & Eq.5 & Eq.6 & Eq.7 & Eq.8 \\
\hline \multirow[t]{3}{*}{ Constante } & $-0,1945$ & $-0,1964$ & $-0,1678$ & $-0,2311$ & $-0,1951$ & 0,0532 & $-0,1508$ & $-0,2311$ \\
\hline & $(0,5443)$ & $(0,5168)$ & $(0,3442)$ & $(0,3046)$ & $(0,2472)$ & $(0,0502)$ & $(0,2580)$ & $(0,3046)$ \\
\hline & {$[-0,3574]$} & {$[-0,3800]$} & {$[-0,4874]$} & {$[-0,7589]$} & {$[-0,7891]$} & {$[1,0595]$} & {$[-0,5847]$} & {$[-0,7589]$} \\
\hline \multirow[t]{3}{*}{$\mathrm{d}(\mathrm{e})_{\mathrm{t}-1}$} & 0,5104 & $-0,0255$ & $-0,8265$ & $-0,4568$ & $-0,6741$ & $-0,4766$ & $-0,6948$ & $-0,4568$ \\
\hline & $(2,3069)$ & $(2,0818)$ & $(1,2704)$ & $(1,1981)$ & $(1,0943)$ & $(0,9566)$ & $(1,1106)$ & $(1,1981)$ \\
\hline & {$[0,2212]$} & {$[-0,0123]$} & {$[-0,6506]$} & {$[-0,3813]$} & {$[-0,6160]$} & {$[-0,4982]$} & {$[-0,6256]$} & {$[-0,3813]$} \\
\hline \multirow[t]{3}{*}{$E(\pi)_{t-1}$} & 0,1005 & 0,1412 & $0,1508^{* *}$ & $0,1757^{* * *}$ & $0,1725^{* * *}$ & $0,1157^{* * *}$ & $0,1536^{* *}$ & $0,1757^{* * *}$ \\
\hline & $(0,1775)$ & $(0,1592)$ & $(\mathrm{o}, 0717)$ & $(0,0581)$ & $(0,0540)$ & $(0,0422)$ & $(\mathrm{o}, 0667)$ & $(0,0581)$ \\
\hline & {$[0,5662]$} & {$[0,8868]$} & {$[2,1036]$} & {$[3,0259]$} & {$[3,1929]$} & {$[2,7427]$} & {$[2,3022]$} & {$[3,0259]$} \\
\hline \multirow[t]{3}{*}{$\pi_{t-1}$} & $0,9755^{* * *}$ & $0,9507^{* * *}$ & $0,9417^{* * *}$ & $0,8815^{* * *}$ & $0,8779^{* * *}$ & $0,8799^{* * *}$ & $0,8806^{* * *}$ & $0,8815^{* * *}$ \\
\hline & $(0,1322)$ & $(0,1175)$ & $(0,0715)$ & $(0,0362)$ & $(0,0341)$ & $(0,0293)$ & $(0,0333)$ & $(0,0362)$ \\
\hline & {$[7,3794]$} & {$[8,0931]$} & {$[13,1667]$} & {$[24,3809]$} & {$[25,7747]$} & {$[30,0777]$} & {$[26,4195]$} & {$[24,3809]$} \\
\hline \multirow[t]{3}{*}{$\mathrm{d}(\mathrm{dlsp})_{\mathrm{t}-1}$} & $-0,7703$ & 0,0903 & & & & & $-0,0496$ & \\
\hline & $(1,4529)$ & $(0,8744)$ & & & & & $(0,4240)$ & \\
\hline & {$[-0,5302]$} & {$[0,1033]$} & & & & & {$[-0,1170]$} & \\
\hline
\end{tabular}




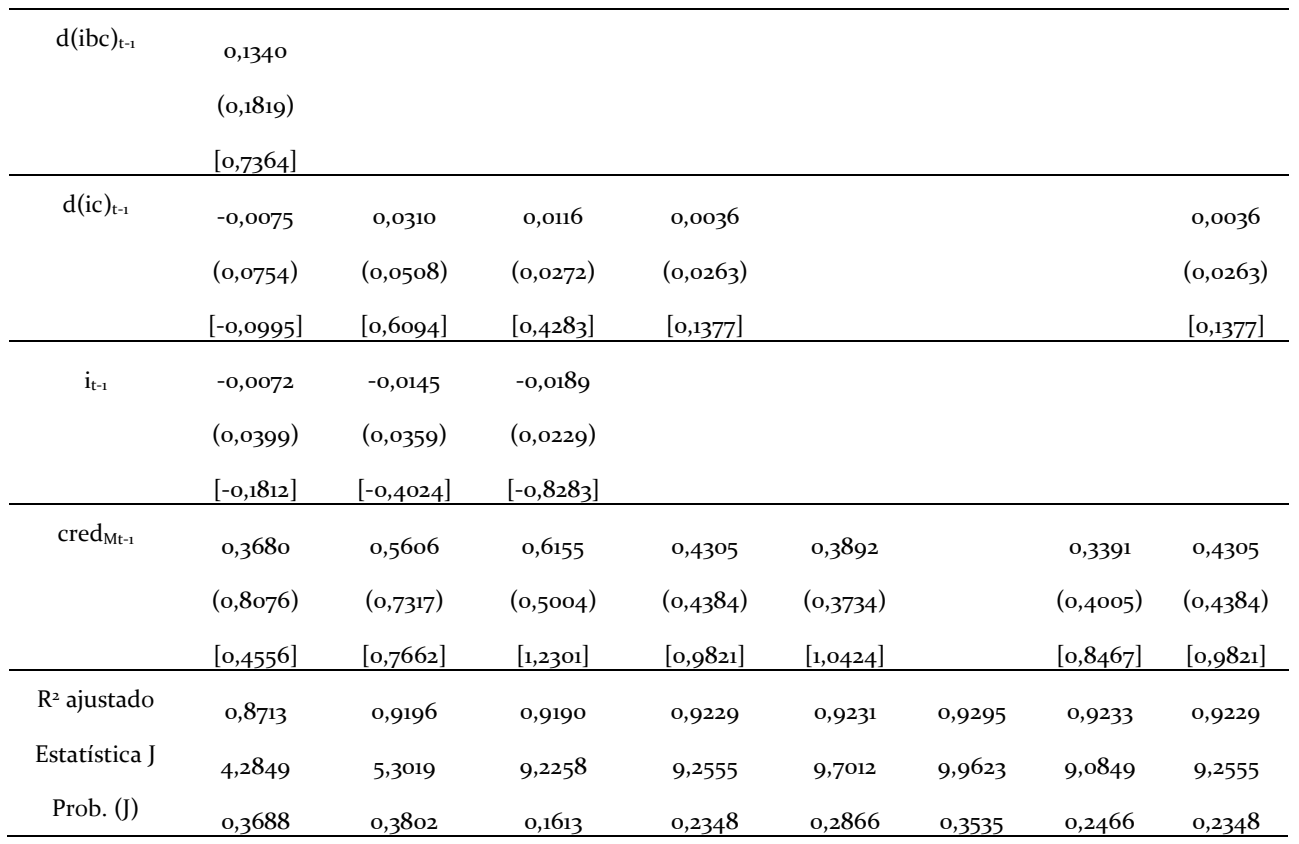

Obs.: ( ) para desvio padrão e [ ] para estatística t. $\left(^{*}\right)$ nível de significância de $10 \%,\left({ }^{* *}\right) 5 \%$ e (***) $1 \%$. As variáveis instrumentais são $E(\mathrm{p})_{t-2}, d(e)_{t-2}, \mathrm{i}_{\mathrm{t}-2}, \mathrm{~d}(\mathrm{ic})_{\mathrm{t}-2}, E(\mathrm{p})_{t-3}, d(e)_{t-3}, \mathrm{i}_{\mathrm{t}-3}, \mathrm{~d}(\mathrm{ic})_{\mathrm{t}-3}, E(\mathrm{p})_{t-4}, d(e)_{t-4}$, $\mathrm{i}_{\mathrm{t}-4}, \mathrm{~d}(\mathrm{ic})_{\mathrm{t}-4}$. Fonte: Elaboração própria.

A partir dos resultados obtidos, identificou-se a continuidade de uma elevada persistência dos desvios inflacionários, com coeficiente variando de o,87 a o,99 e todos significantes estatisticamente a $1 \%$, nos dois casos testados, com cred $_{\mathrm{CK}}$ e $\operatorname{cred}_{\mathrm{M}}$. Por sua vez, o coeficiente associado ao papel dos desvios das expectativas de inflação apresentou valores de o,10 a 0,46 e com significância variando de $10 \%$ a 1\%.

As variações cambiais não apresentaram papel significante estatisticamente sobre os desvios de inflação, e o sinal estimado foi, na maior parte, negativo e em contraste com o previsto. Portanto, pelas estimações GMM o efeito pass-through sobre os desvios de inflação não foi corroborado em nenhuma das especificações.

Em relação à variação do $i b c$, do ic e da $d l s p$, assim como ao papel da Selic, nenhum desses mostrou correlação estatisticamente significante com os desvios inflacionários. Por fim, a credibilidade da política monetária, por meio do cred $\mathrm{d}_{\mathrm{CK}} \mathrm{e}$ do $\operatorname{cred}_{\mathrm{M}}$, não demonstrou relevância na explicação do processo inflacionário, além dos coeficientes estimados terem sinal positivo, em divergência com o previsto. Uma forma de explicar este tipo de puzzle poderia ser pela contrapartida de uma alta persistência dos desvios inflacionários, que foi captada pelo $\alpha_{3}$. Tal persistência poderia estar rompendo com o canal de transmissão da credibilidade para a 
inflação, previsto pela literatura, resultando em uma correlação positiva entre ambas as variáveis. Nas regressões por GMM, o teste J, para todas as regressões, confirmou a hipótese nula de correta especificação dos modelos.

3.4. Índice de commodities do FMI, taxa de desemprego e os desvios inflacionários

Para fins de verificação de robustez das estimações preliminares por GMM em particular, as variáveis $\mathrm{d}(\mathrm{ic})$ e $\mathrm{d}(\mathrm{ibc})$ foram substituídas, respectivamente, por $\mathrm{d}(\mathrm{icfmi})$ e u nos dois casos adotados de índices de credibilidade, além de algumas estimações que foram realizadas com a ausência de constante. As Tabelas 7 e 8 mostram os resultados obtidos.

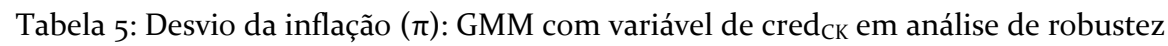

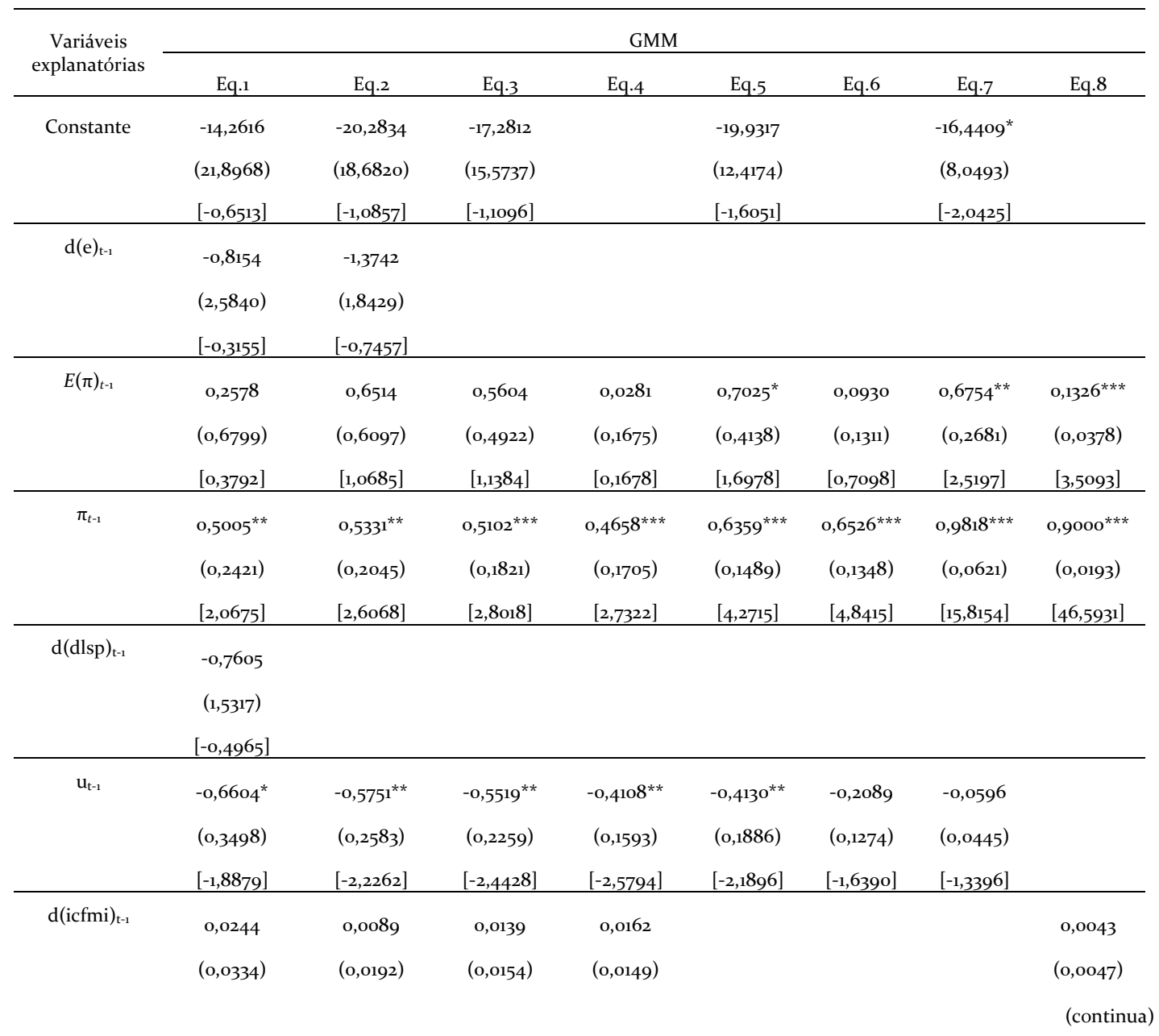


(continuação)

\begin{tabular}{|c|c|c|c|c|c|c|c|c|}
\hline & {$[0,7312]$} & {$[0,4642]$} & {$[0,8999]$} & {$[1,0871]$} & & & & {$[0,9158]$} \\
\hline \multirow[t]{3}{*}{$\mathrm{i}_{\mathrm{t}-1}$} & $0,3306^{*}$ & $0,3025^{* *}$ & $0,2919^{* *}$ & $0,2329^{* *}$ & $0,2065^{*}$ & 0,1101 & & \\
\hline & $(0,1995)$ & $(0,1523)$ & $(0,1273)$ & $(0,0980)$ & $(0,1106)$ & $(0,0788)$ & & \\
\hline & {$[1,6575]$} & {$[1,9864]$} & {$[2,2938]$} & {$[2,3766]$} & {$[1,8679]$} & {$[1,3970]$} & & \\
\hline \multirow[t]{3}{*}{$\operatorname{cred}_{\mathrm{CK} \mathrm{t}-1}$} & 16,3580 & 22,1451 & 19,0123 & 0,8924 & 21,5428 & o,6095 & $17,2991^{*}$ & \\
\hline & $(23,0685)$ & $(19,6724)$ & $(16,4398)$ & $(0,6836)$ & $(13,0768)$ & $(0,4995)$ & $(8,4021)$ & \\
\hline & {$[0,7091]$} & {$[1,1257]$} & {$[1,1565]$} & {$[1,3055]$} & {$[1,6474]$} & {$[1,2202]$} & {$[2,0589]$} & \\
\hline $\mathrm{R}^{2}$ ajustado & 0,6898 & o,686o & o,7076 & o,80o8 & 0,7722 & 0,8904 & 0,8923 & 0,9306 \\
\hline Estatística J & 2,2185 & 2,8620 & 3,9445 & 5,8835 & 4,5601 & 6,4954 & 6,6226 & 9,9048 \\
\hline Prob. (J) & o,6957 & 0,7213 & 0,6842 & 0,5534 & 0,7135 & 0,5919 & o,5778 & 0,4489 \\
\hline
\end{tabular}

Obs.: ( ) para desvio padrão e [ ] para estatística t. $\left({ }^{*}\right)$ nível de significância de $10 \%,\left({ }^{* *}\right) 5 \%$ e $\left({ }^{* *}\right)$ 1\%. As variáveis instrumentais são $E(\mathrm{p})_{t-2}, d(e)_{t-2}, \mathrm{i}_{\mathrm{t}-2}, \mathrm{~d}(\mathrm{icfmi})_{\mathrm{t}-2}, E(\mathrm{p})_{t-3}, d(e)_{t-3}, \mathrm{i}_{\mathrm{t}-3}, \mathrm{~d}(\mathrm{icfmi})_{\mathrm{t}-3}, E(\mathrm{p})_{t-4}$, $d(e)_{t-4}, \mathrm{i}_{\mathrm{t}-4}, \mathrm{~d}(\mathrm{icfmi})_{\mathrm{t}-4}$. Fonte: Elaboração própria.

Tabela 6: Desvio da inflação $(\pi)$ : GMM com variável de cred $_{M}$ em análise de robustez

\begin{tabular}{|c|c|c|c|c|c|c|c|c|}
\hline \multirow{2}{*}{$\begin{array}{c}\text { Variáveis } \\
\text { explanatórias }\end{array}$} & \multicolumn{8}{|c|}{ GMM } \\
\hline & Eq.1 & Eq.2 & Eq.3 & Eq.4 & Eq.5 & Eq.6 & Eq.7 & Eq.8 \\
\hline \multirow[t]{3}{*}{ Constante } & 1,0333 & 0,4557 & 0,5172 & & 0,2551 & & $-0,2178^{* *}$ & \\
\hline & $(0,9495)$ & $(0,7396)$ & $(0,7199)$ & & $(0,6097)$ & & $(0,3675)$ & \\
\hline & {$[1,0883]$} & {$[0,6161]$} & {$[0,7184]$} & & {$[0,4183]$} & & {$[-0,5927]$} & \\
\hline \multirow[t]{3}{*}{$d(e)_{t-1}$} & $-0,8450$ & $-1,3024$ & & & & & & \\
\hline & $(2,5895)$ & $(1,8466)$ & & & & & & \\
\hline & {$[-0,3263]$} & {$[-0,7053]$} & & & & & & \\
\hline \multirow[t]{3}{*}{$E(\pi)_{t-1}$} & $-0,2570$ & $-0,0546$ & $-0,0504$ & o,0956 & o,0094 & 0,0881 & 0,1292 & $0,1326^{* * *}$ \\
\hline & $(0,3875)$ & $(0,2418)$ & $(0,2502)$ & $(0,1797)$ & $(0,2276)$ & $(0,1679)$ & $(0,1021)$ & $(0,0378)$ \\
\hline & {$[-0,6631]$} & {$[-0,2258]$} & {$[-0,2013]$} & {$[0,5320]$} & {$[0,0414]$} & {$[0,5250]$} & {$[1,2658]$} & {$[3,5093]$} \\
\hline \multirow[t]{3}{*}{$\pi_{t-1}$} & $0,5096^{* *}$ & $0,5351^{* *}$ & $0,5147^{* * *}$ & $0,6011^{* * *}$ & $0,6362^{* * *}$ & $0,6781^{* * *}$ & $0,9829^{* * *}$ & $0,9000^{* * *}$ \\
\hline & $(0,2434)$ & $(0,2104)$ & $(0,1863)$ & $(0,1623)$ & $(0,1538)$ & $(0,1557)$ & $(0,0618)$ & $(\mathrm{o}, 0193)$ \\
\hline & {$[2,0935]$} & {$[2,5435]$} & {$[2,7633]$} & {$[3,7029]$} & {$[4,1376]$} & {$[4,3556]$} & {$[15,9062]$} & {$[46,5931]$} \\
\hline \multirow[t]{3}{*}{$\mathrm{d}(\mathrm{dlsp})_{\mathrm{t}-1}$} & $-0,6935$ & & & & & & & \\
\hline & $(1,5673)$ & & & & & & & \\
\hline & {$[-0,4425]$} & & & & & & & \\
\hline $\mathrm{u}_{\mathrm{t}-1}$ & $-0,6737^{*}$ & $-0,6068^{* *}$ & $-0,5845^{* *}$ & $-0,4668^{* *}$ & $-0,4476^{* *}$ & $-0,3862^{*}$ & $-0,0832$ & \\
\hline
\end{tabular}




\begin{tabular}{|c|c|c|c|c|c|c|c|c|}
\hline & $(0,3688)$ & $(0,2739)$ & $(0,2408)$ & $(0,2231)$ & $(0,2056)$ & $(0,2051)$ & $(0,0532)$ & \\
\hline & {$[-1,8265]$} & {$[-2,2153]$} & {$[-2,4275]$} & {$[-2,0921]$} & {$[-2,1775]$} & {$[-1,8827]$} & {$[-1,5642]$} & \\
\hline \multirow[t]{3}{*}{$\mathrm{d}(\mathrm{icfmi})_{\mathrm{t}-1}$} & 0,0234 & o,0093 & 0,0140 & 0,0115 & & & & 0,0043 \\
\hline & $(0,0338)$ & $(0,0193)$ & $(0,0154)$ & $(0,0129)$ & & & & $(0,0047)$ \\
\hline & {$[0,6932]$} & {$[0,4827]$} & {$[0,9106]$} & {$[0,8892]$} & & & & {$[0,9158]$} \\
\hline \multirow[t]{3}{*}{$\mathrm{i}_{\mathrm{t}-1}$} & 0,3258 & $0,3025^{* *}$ & $0,2938^{* *}$ & $0,2348^{*}$ & $0,2087^{*}$ & 0,1782 & & \\
\hline & $(0,1994)$ & $(0,1529)$ & $(0,1280)$ & $(0,1302)$ & $(0,1135)$ & $(0,1208)$ & & \\
\hline & {$[1,6340]$} & {$[1,9792]$} & {$[2,2950]$} & {$[1,8027]$} & {$[1,8382]$} & {$[1,4749]$} & & \\
\hline \multirow[t]{3}{*}{$\operatorname{cred}_{\mathrm{M} t-1}$} & 1,2784 & 1,7388 & 1,5338 & $1,8037^{*}$ & 1,6848 & $1,7986^{*}$ & $1,3053^{*}$ & \\
\hline & $(1,8654)$ & $(1,5391)$ & $(1,3046)$ & $(1,0835)$ & $(1,0601)$ & $(0,9851)$ & $(0,7058)$ & \\
\hline & {$[0,6853]$} & {$[1,1298]$} & {$[1,1757]$} & {$[1,6648]$} & {$[1,5893]$} & {$[1,8259]$} & {$[1,8495]$} & \\
\hline $\mathrm{R}^{2}$ ajustado & o,6940 & 0,6809 & 0,7011 & 0,7366 & 0,7672 & 0,7798 & o,8914 & o,9306 \\
\hline Estatística J & 2,0429 & 2,6426 & 3,6125 & 3,9881 & 4,2346 & 4,3984 & 6,2487 & 9,9048 \\
\hline Prob. (J) & 0,7279 & 0,7549 & 0,7289 & 0,7812 & 0,7524 & 0,8195 & 0,6194 & 0,4489 \\
\hline
\end{tabular}

Obs.: ( ) para desvio padrão e [ ] para estatística t. $\left({ }^{*}\right)$ nível de significância de $10 \%,\left({ }^{* *}\right) 5 \%$ e $\left({ }^{* * *}\right)$ 1\%. As variáveis instrumentais são $E(\mathrm{p})_{t-2}, d(e)_{t-2}, \mathrm{i}_{\mathrm{t}-2}, \mathrm{~d}(\mathrm{icfmi})_{\mathrm{t}-2}, E(\mathrm{p})_{t-3}, d(e)_{t-3}, \mathrm{i}_{\mathrm{t}-3}, \mathrm{~d}(\mathrm{icfmi})_{\mathrm{t}-3}, E(\mathrm{p})_{t-4}$, $d(e)_{t-4}, \mathrm{i}_{\mathrm{t}-4}, \mathrm{~d}(\mathrm{icfmi})_{\mathrm{t}-4}$. Fonte: Elaboração própria.

O resultado mais interessante com as substituições mencionadas diz respeito ao papel da taxa de desocupação na determinação dos desvios inflacionários, que converge com o esperado pela Curva de Phillips na maior parte das especificações. O sinal é negativo e significativo estatisticamente, variando de $10 \%$ a $1 \%$ de confiança. Ademais, para os casos em que a taxa de desocupação mostrou significância, o coeficiente $\alpha_{3}$ apresentou valores menores $(0,44$ a 0,67$)$ do que os estimados previamente $(0,87$ a 0,99$)$.

Um outro resultado diferente face às estimações anteriores foi a significância estatística encontrada para a Selic, na maior parte das especificações para os dois índices de credibilidade. No entanto, o coeficiente estimado é positivo. Embora em contraste com a intuição econômica, isso possivelmente se deve à existência de defasagens nos efeitos da política monetária, de modo que uma elevação da taxa Selic é acompanhada no mês seguinte por um aumento do desvio inflacionário, dada sua inércia parcial e enquanto os efeitos integrais da política monetária não são efetivados.

O índice de commodities do FMI não mostrou impactos sobre os desvios inflacionários, corroborando os resultados encontrados com o índice do BCB (IC$\mathrm{Br}$ ). Isso sugere que no período estudado os desvios inflacionários não podem ser 
creditados a condicionantes externos, tais como a dinâmica dos preços de commodities e das variações cambiais.

Em relação aos índices de credibilidade, todos eles apresentaram significância estatística em alguns casos, porém com sinal positivo. Da mesma forma que mencionado para as estimações anteriores, possivelmente o sinal positivo dos coeficientes relacionados à credibilidade deve-se à presença de persistência dos desvios inflacionários, ainda que no caso das estimações com a taxa de desocupação tal inércia seja menor. Além disto, nos casos em que a taxa de desocupação é significativa estatisticamente os coeficientes associados ao papel dos desvios das expectativas de inflação não apresentam significância estatística. Isto poderia sugerir uma ausência de ancoragem das expectativas inflacionárias nas metas anunciadas pelo $\mathrm{BCB}$, de modo que os desvios inflacionários se tornam mais backward-looking. Por fim, todas as estimações confirmaram, com base no teste J, a hipótese de correta especificação.

\subsection{Defasagens temporais e respostas ao impulso por modelos VAR Bayesianos}

Com base nas estimações realizadas algumas variáveis apontadas como relevantes pela literatura teórica deixam de explicar ou explicam pouco os desvios inflacionários no Brasil recente. Como argumentado, esse tipo de puzzle pode ser devido à elevada persistência estimada para os desvios inflacionários, i.e. ao padrão eminentemente backward-looking de tais desvios. Contudo, podem-se colocar sob suspeita tais resultados pelo fato de que algumas variáveis, tais como a taxa básica de juros, as variações cambiais e mesmo a credibilidade da política monetária, requerem defasagens temporais expressivas para terem seus efeitos sobre os preços fixados pelas firmas. Portanto, para fins de possível contorno dessa limitação nas estimações conduzidas por GMM, realizamos experimentos por VAR Bayesiano (BVAR) com as mesmas variáveis, dando-se atenção especial às funções impulsoresposta generalizadas, destacando-se as respostas de $\pi$ a impulsos gerados sobre as demais variáveis.

Deve-se salientar que no processo de calibração do BVAR os priors foram ajustados e configurados seguindo o padrão apontado pela literatura relacionada (Kadiyala e Karlsson, 1997; Chauvet e Potter, 2012). O método Litterman/Minnesota é composto de quatro priors: $\mu_{1}, \lambda_{1}, \lambda_{2}$ e $\lambda_{3}$ : $\mu_{1}$ é o prior médio e foi configurado nas estimações com valor igual a zero, para diminuir o risco de sobreajuste (overfitting) do modelo; $\lambda_{1}$ é a restrição sobre a variância (overall tightness) e controla a importância relativa da amostra e da informação a priori. Definimos esse prior como o,1 para permitir que a informação a priori domine a informação amostral; $\lambda_{2}$ representa a restrição da variância das outras variáveis. Definimos esse prior 
como o,99; por fim, $\lambda_{3}$ representa o padrão de decaimento do modelo e o definimos como 1,o para impor decaimento linear (linear decay).

Basicamente, implementaram-se dois modelos BVAR. O primeiro modelo com as seguintes variáveis: $\pi, \mathrm{E}(\pi), \mathrm{d}(\mathrm{icfmi}), \mathrm{d}(\mathrm{dlsp}), \mathrm{u}, \mathrm{cred}_{\mathrm{CK}}, \mathrm{d}(\mathrm{e}), \mathrm{i}$, respeitandose as respectivas ordens de integração das séries temporais, enquanto o segundo

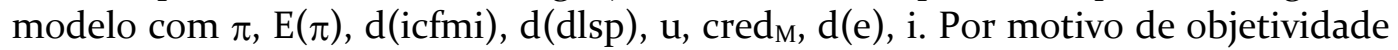
não serão apresentados os resultados do modelo BVAR com d(ibc) e d(ic), visto que isso tornaria a apresentação dos resultados muito exaustiva. Além disso, as análises por impulso-resposta foram implementadas pelo método generalizado, que é robusto a mudanças de ordenamento das séries, como apontado por Koop et. al. (1996) e Pesaran e Shin (1998).

Para definição do número ótimo de lags do BVAR, identificaram-se inicialmente os critérios de informação de Akaike, Schwarz e Hannan-Quinn, assim como os devidos testes de auto-correlação $L M$ e de estabilidade dos parâmetros por meio das raízes inversas do polinômio característico (Apêndice) para um VAR irrestrito e, em seguida, o número ótimo de lags foi adotado para os modelos BVAR com as mesmas séries temporais. Tais critérios e testes demonstraram que o BVAR deveria ter o6 lags para as duas configurações ( cred $_{\text {ск }}$ e $\operatorname{cred}_{M}$ ) de modo que estimamos um BVAR (6) nesses dois casos.

O Gráfico 2 apresenta respostas generalizadas ao impulso a partir do BVAR (6) com $_{\text {cred }}{ }_{C K}$. De fato, a partir do BVAR estimado observa-se que, ao contrário dos resultados por GMM, os desvios inflacionários apresentam relativa sensibilidade a variáveis que não apresentaram relevância nas estimações por estes dois últimos métodos. Em primeiro lugar, um choque positivo na credibilidade, medida pelo cred $_{\mathrm{CK}}$, implicou desvios inflacionários menores que os valores normais ao longo de 24 meses após o referido choque, sendo que o valor mínimo ocorreu aproximadamente o8 meses após o mesmo choque. Por sua vez, os efeitos de um aumento na taxa Selic sobre os desvios inflacionários, tal como esperado, demoram algum tempo para se efetivarem. Apenas após 12 meses do choque positivo na Selic há desvios inflacionários menores que os valores normais.

Por outro lado, embora a partir de dez meses após um choque em $d(e)$ seja verificada uma elevação dos desvios inflacionários acima dos valores normais, tal elevação é muito sutil, o que demonstra um efeito pass-through fraco ou menor do que o sugerido pela literatura para o país (de Mendonça e Tostes, 2015). Já os choques positivos em $d(d l s p)$ estão associados a efeitos de redução dos desvios inflacionários, o que contradiz a hipótese de teoria fiscal do nível de preços, segundo a qual uma elevação da dívida pública está associada a efeitos inflacionários como mecanismo de ajuste e satisfação da restrição orçamentária do governo (Woodford, 2001). 
Choques positivos na taxa de desemprego parecem não ter efeito expressivo sobre os desvios inflacionários. De fato, tais choques vêm acompanhados de desvios inflacionários maiores que os normais, situação que se inverte apenas após 20 meses de ocorrido o choque na taxa de desemprego. Esses resultados também representam uma diferença em relação às estimações por GMM, e podem ser interpretadas como um momento de estagflação, visto que os desvios inflacionários estariam aumentando por meses, mesmo após a elevação observada na taxa de desemprego. Além disso, tal situação também pode ser interpretada como uma contrapartida de elevada persistência dos desvios inflacionários estimados. Essa persistência também está presente nas respostas dos desvios de inflação após um impulso na própria variável $(\pi)$. Mesmo após 24 meses de ocorrido o choque em $\pi$, esta continua a apresentar valores maiores que os esperados, o que denota elevada persistência.

No entanto, os resultados pelo BVAR estimado dão peso maior ao papel das expectativas do público, em comparação com os coeficientes estimados por GMM. Pode-se verificar que imediatamente após um choque em $\mathrm{E}(\pi)$, os desvios inflacionários sofrem uma elevação e convergem gradualmente para seus valores normais ao longo de 24 meses. Isso significa que o componente backward-looking é menor nas estimações pelo BVAR, que permitem uma análise mais adequada em termos de defasagens temporais entre impulsos e respostas. Finalmente, choques positivos em $d$ (icfmi) estão associados a efeitos de aumento em $\pi$ acima dos valores normais, apesar de tais efeitos não serem muito expressivos em relação aos efeitos a partir de choques em $\mathrm{E}(\pi), \pi$ e cred ${ }_{\text {Ск. }} \mathrm{O}$ efeito de transmissão inflacionária a partir de commodities é esperado para uma economia exportadora-líquida desses bens, tais como o Brasil (Moreira, 2014). Contudo, ao considerarmos os resultados como um todo, não é possível afirmar que condicionantes externos, tais como variações cambiais e de preços de commodities, sejam creditados como fontes principais dos desvios inflacionários no país.

Quando estimamos o BVAR com uso do índice $\operatorname{cred}_{M}$ para medir a credibilidade da política monetária, chega-se a respostas aos impulsos muito semelhantes às do modelo anterior. O Gráfico 3 mostra as novas funções. Pode-se observar que de modo geral não há mudança significativa nas relações estimadas. $\mathrm{O}$ papel da credibilidade sofre uma redução quando medida pelo cred $_{\mathrm{M}}$, porém ainda assim choques positivos nessa variável estão associadas a desvios inflacionários menores que os esperados ao longo de 24 meses. 


\section{Gráfico 2 - Respostas generalizadas ao impulso a partir do BVAR(6) com cred CK $_{\mathrm{C}}$}

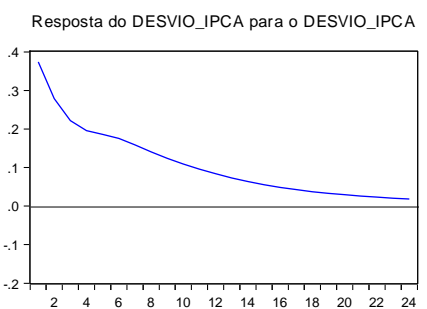

Resposta do DESVIO_IPCA para o D(DLSP)

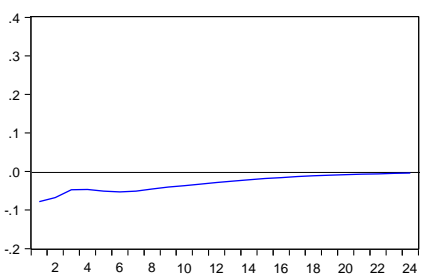

Resposta do DESVIO_IPCA para o D(ICFMI)

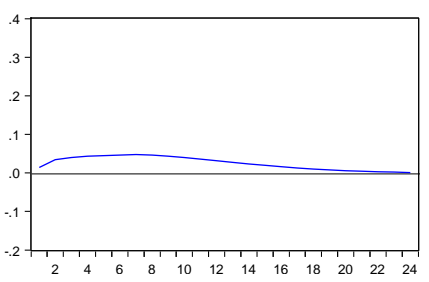

Resposta do DESVIO_IPCA para o DESVIO_EXP_IPCA

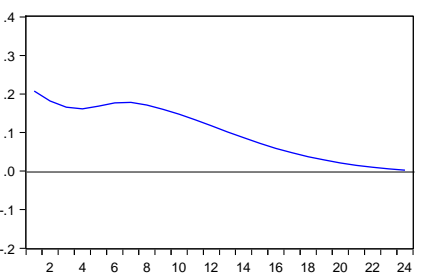

Resposta do DESVIO_IPCA para o CREDCK

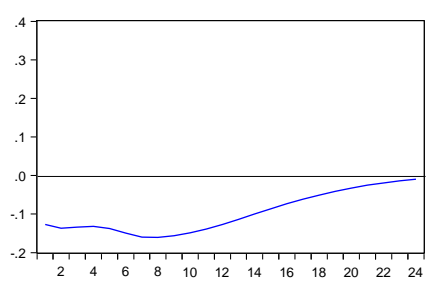

Resposta do DESVIO_IPCA para o U

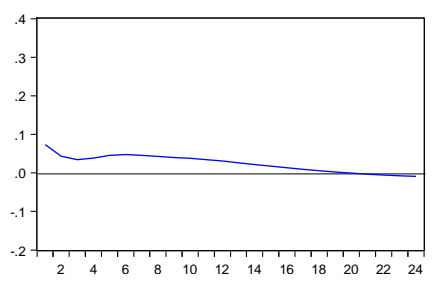

Fonte: Elaboração própria.
Resposta do DESVIO_IPCA para o D(E)

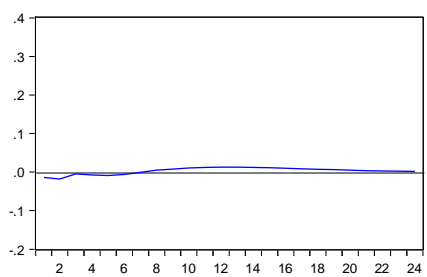

Resposta do DESVIO_IPCA para o I

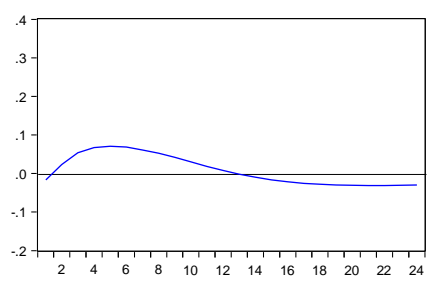




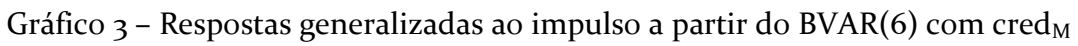

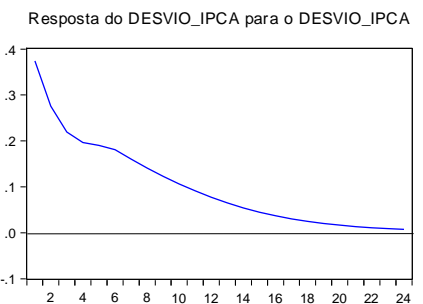

Resposta do DESVIO_IPCA para o D(DLSP)

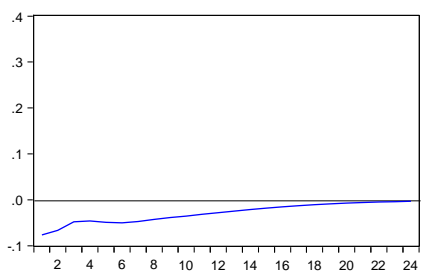

Resposta do DESVIO_IPCA para o D(ICFMI)

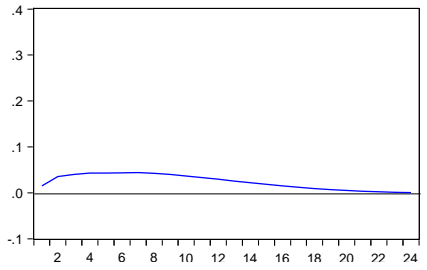

Resposta do DESVIO_IPCA para o DESVIO_EXP_IPCA

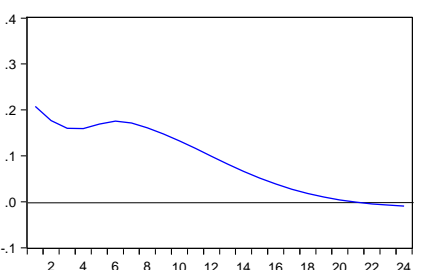

Resposta do DESVIO_IPCA para o CREDM

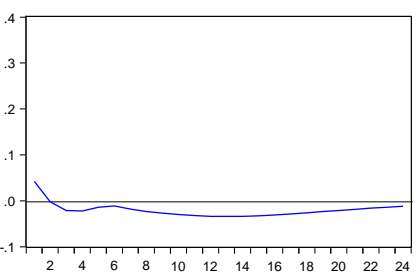

Resposta do DESVIO_IPCA para o U

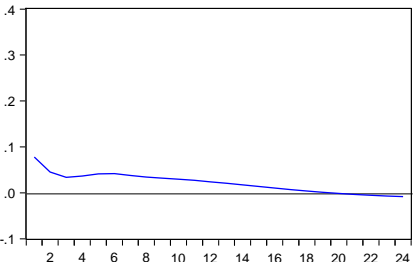

Fonte: Elaboração própria.

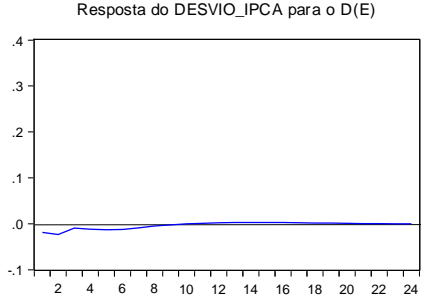

Resposta do DESVIO_IPCA para o I

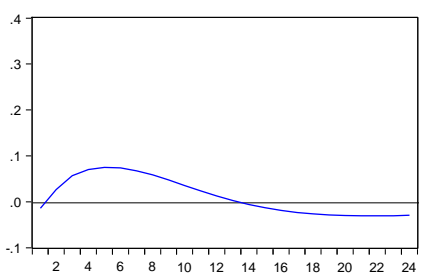

\section{Conclusões}

O presente trabalho identificou o grau de inércia ou persistência dos desvios inflacionários no Brasil, além dos fatores determinantes de tais desvios, no período de janeiro de 2003 a dezembro de 2014. Em relação ao grau de persistência dos desvios, as estimações por GMM demonstraram um coeficiente significativo estatisticamente e com valor no intervalo de o,87 a o,99 nas estimações com d(ic) e $\mathrm{d}(\mathrm{ibc})$, e de o,44 a o,67 nas estimações com d(icfmi) e taxa de desocupação (u), sugerindo que os desvios inflacionários na economia brasileira recente são mais sensíveis, do ponto de vista do lado real doméstico, ao comportamento do mercado de trabalho e da taxa de desemprego, se comparado com o papel desempenhado pela dinâmica do produto ou do PIB. Todavia, nas estimações de respostas generalizadas ao impulso pelos modelos BVAR, identificou-se a relativa resistência dos desvios inflacionários a elevações na taxa de desemprego. 
As estimações por GMM apontaram para uma dinâmica backward-looking dos desvios inflacionários, ou seja, o processo de determinação de tais desvios estaria mais ancorado em sua dinâmica passada do que nas expectativas do público a respeito dos desvios futuros. No entanto, o teor backward-looking dos desvios inflacionários foi relativamente atenuado com as estimações pelos modelos BVAR. Por sua vez, embora os índices usados como proxy da credibilidade não tenham confirmado a teoria de que maiores níveis de credibilidade seriam acompanhados de menores taxas de inflação ou de desvios inflacionários nas estimações por GMM, essa "falha" foi corrigida pelas funções impulso-resposta dos modelos BVAR, que confirmaram aquela teoria, com maior peso para o $\mathrm{cred}_{C K}$.

Deve-se mencionar que as diferenças de resultados entre os métodos GMM e BVAR são devidas ao fato de que neste último as relações de resposta ao impulso são geradas a partir de um sistema de equações em que todas as variáveis são endógenas em relação às demais, assim como ao fato de que no BVAR os coeficientes são estimados como distribuições probabilísticas, o que dá a esse método maior robustez em relação àqueles dois primeiros. Outros fatos estilizados também foram confirmados pelas estimações BVAR, tais como os efeitos com defasagens temporais expressivas a partir de aumentos na taxa Selic e os impactos a partir de elevações nos preços de commodities, muito embora os efeitos via passthrough da taxa de câmbio sejam, ao que tudo indica, menos relevantes do que o sugerido pela literatura. É importante frisar que estes condicionantes externos (câmbio e preços de commodities) não poderiam ser considerados como fontes relevantes de explicação dos desvios inflacionários no período estudado. Ao que tudo indica as fontes maiores para os desvios estão localizados em fatores domésticos.

\section{Referências}

BALL, L. Efficient rules for monetary policy, International Finance, v. 2, n. 1, p. 63-83, 1999. DOI: https://doi.org/10.1111/1468-2362.00019

BALL, L. Policy rules for open economy, NBER, p. 127-156, 1999b.

BARRO, R. J.; GORDON, D. B. Rules, discretion and reputation in a model of monetary policy, Journal of Monetary Economics, v. 12, n. 1, p. 101-121, jul. 1983. DOI: https://doi.org/10.1016/0304-3932(83)g0051-X

CAMPÊLO, A. K. \& CRIBARI-NETO, F. Inflation Inertia and Inliers: The Case of Brazil. Revista Brasileira de Economia, v. 57, n. 4, p. 713-739, out./dez. 2003. DOI: https://doi.org/10.1590/So034-71402003000400003 
CATI, R. C.; GARCIA, M. G. P. \& PERRON, P. Unit Roots in the Presence of Abrupt Governmental Interventions with an Application to Brazilian Data. Journal of Applied Econometrics, v. 14, p. 27-56, 1999. DOI: https://doi.org/10.1002/(SICI)1099$1255(199901 / 02) 14: 1<27:: A I D-J A E 490>3.0 . C O ; 2-G$

CECCHETTI, S.; KRAUSE, S. Central Bank structure, policy efficiency and macroeconomic performance: Exploring empirical relationships, Federal Reserve Bank of St. Louis Economic Review, v. 84, p.47-59, 2002.

CERISOLA, M.; GELOS, G. What drives inflation expectations in Brazil? An empirical analysis, Applied Economics, v. 41, n. 10, p. 1215-1227, 2009. DOI: https://doi.org/10.1080/00036840601166892

CHAUVET, M.; POTTER, S. Forecasting Output, Handbook of Economic Forecasting, v. 2, 2012.

CLARIDA, R.; GALÍ, J.; GERTLER, M. The science of monetary policy: a new Keynesian perspective, Journal of Economic Literature, v. 37, p. 1661-1707, dez. 1999. DOI: https://doi.org/10.1257/jel.37.4.1661

CRAGG, J. G. More efficient estimation in the presence of heteroscedasticity of unknown form, Econometrica, v. 51, n. 3, p. 751-763, maio. 1983.

CYSNE, R. P. A Relação Phillips no Brasil: 1964-66 x 1980-1984. Revista Brasileira de Economia, v. 39, n. 4, p. 401-422, out./dez. 1985.

DUREVALL, D. The dynamics of chronic inflation in Brazil, 1968-1985, Journal of Business and Economic Statistics, v. 16, n. 4, p. 423-432, out. 1998.

FIGUEIREDO, E. A.; MARQUES, A. M. Inflação Inercial como um Processo de Longa Memória: Análise a partir de um Modelo ARFIMA-FIGARCH, Estudos Econômicos, v. 39, n. 2, p. 437-458, abr./jun. 2009. DOI: https://doi.org/10.1590/So101-41612009000200008

FIGUEIREDO, E. A. \& MARQUES, A. M. Inflação inercial sob mudanças de regime: análise a partir de um modelo MS-ARFIMA, 1944-2009, Economia Aplicada, v. 15, n. 3, p. 443-457, 2011. DOI: https://doi.org/10.1590/S1413-80502011000300005

GOMES DA SILVA, C. \& LEME, M.C.S. An Analysis of the Degrees of Persistence of Inflation, Inflation Expectations and Real Interest Rate in Brazil. Revista Brasileira de Economia, v. 65, n. 3, p. 289-302, jul./set. 2011. DOI: https://doi.org/10.1590/So03471402011000300004 
GOMES DA SILVA, C. \& VIEIRA, F. V. Persistência Inflacionária Regional Brasileira: Uma Aplicação dos Modelos ARFIMA. Economia Aplicada, v. 17, n. 1, p. 115-134, 2013. DOI: https://doi.org/10.1590/S1413-80502013000100006

GOTTSCHALK, R. Testing for breaks in inertia: An alternative approach, Applied Economics Letters, v. 10, n. 3, p. 161-163, fev. 2003. DOI: https://doi.org/10.1080/1350485022000044048

HOLANDA BARBOSA, F. de; MCNELIS, P. D. Indexation and inflationary inertia: Brazil 1964 - 1985, World Bank Economic Review, v. 3, n. 3, p. 339-357, 1989. DOI: https://doi.org/10.1093/wber/3.3.339

KADIYLAIA, K. R.; KARLSSON, S. Numerical methods for estimation and inference in Bayesian VAR-models, Journal of Applied Econometrics, v. 12, p. 99-132, mar. 1997. DOI: https://doi.org/10.1002/(SICI)1099-1255(199703)12:2<99::AID-JAE429>3.0.CO;2-A

KOOP, G.; KOROBILIS, D. Bayesian multivariate time series methods for empirical macroeconomics, Foundations and Trends in Econometrics, v. 3, n. 4, p. 267-358, 2009. DOI: https://doi.org/10.1561/08000ooo13

KOOP, G.; PESARAN, M. H.; POTTER, S. M. Impulse response analysis in nonlinear multivariate models, Journal of Econometrics, v. 74, p. 119-147, set. 1996 . DOI: https://doi.org/10.1016/0304-4076(95)01753-4

LITTERMAN, R. Forecasting With Bayesian Vector Autoregressions - Five Years of Experience, Journal of Business and Economic Statistics, v. 4, p. 25-38, 1986.

LUTKENPOHL, H. Introduction to Multiple Time Series Analysis. Berlin: Springer, 1991. DOI: https://doi.org/10.1007/978-3-662-02691-5.

MACHADO, V. G. \& PORTUGAL, M.S. Measuring inflation persistence in Brazil using a multivariate model. Revista Brasileira de Economia, v. 68, n. 2, p. 225-241, abr./jun. 2014. DOI: https://doi.org/10.1590/So034-71402014000200004

MENDONÇA, H. de. Towards credibility from inflation targeting: The Brazilian experience, Applied Economics, v. 39, n. 20, p. 2599-2615, 2007. DOI: https://doi.org/10.1080/00036840600707324

MENDONÇA, H. F. de; TOSTES, F. S. The Effect of Monetary and Fiscal Credibility on Exchange Rate Pass-Through in an Emerging Economy, Open Economies Review, v. 26, n. 4, p. 787-816, set. 2015. DOI: https://doi.org/10.1007/s11079-014-9339-3 
MONTES, G. C.; TIBERTO, B. P. Gestão da dívida pública, reputação fiscal e risco país Evidências empíricas para o Brasil, Planejamento e Política Públicas, n. 44, p. 343-373, 2015.

MOREIRA, R. R. Some notes on a commodity net-exporting economy: exchange rate, consumer inflation and monetary policy, Economics and Finance Review, v. 3, n. 8, p. 26-30, jun. 2014.

NOVAES, A.D. Revisiting the inertial inflation hypothesis for Brazil, Journal of Development Economics, v. 42, n. 1, p. 89-110, 1983. DOI: https://doi.org/10.1016/03043878(93)90074-W

PESARAN, M. H., SHIN, Y. Generalized Impulse Response Analysis in Linear Multivariate Models, Economics Letters, v. 58, p. 17-29, 1998. DOI: https://doi.org/10.1016/So165$1765(97) 00214-0$

PONZONI, G. A.; ZILLI, J. B. Unemployment and Inflation: An Estimated Phillips Curve for Brazil (2002-2014), Journal of Finance and Economics, v. 3, n. 5, p. 77-85, 2015.

REISEN, V. A.; CRIVARI-NETO, F.; JENSEN, M. J. Long memory inflationary dynamics: The case of Brazil, Studies in Nonlinear Dynamics and Econometrics, v. 7, n. 3, out.. 2003. DOI: https://doi.org/10.2202/1558-3708.1157

SVENSSON, L. Inflation forecast targeting: implementing and monitoring inflation targets, European Economic Review, v. 41, p. 1111-1146, 1997. DOI: https://doi.org/10.1016/Soo142921(96)00055-4

VElOSO, G. O.; FEISTEL, P. R.; HOECKEL, P. H. O.; Casagrande, D. L.; Santos, C. A. P. A Curva de Phillips: uma análise da economia brasileira de 2002 a 2012 . Revista Economia \& Tecnologia (RET), v. 9, n. 2, p. 05-19, abr./jun. 2013.

WOODFORD, M. Fiscal requirements for price stability, Journal of Money, Credit and Banking, v. 33, n. 3, p. 669-728, 2001. DOI: https://doi.org/10.2307/2673890

WOOLDRIDGE, J. M. Applications of generalized method of moments estimation. Journal of Economic Perspectives, v. 15, n. 4, p. 87-100, 2001. DOI: https://doi.org/10.1257/jep.15.4.87 


\section{Apêndice}

Tabela Al - Testes LM de autocorrelação residual para o BVAR com cred ${ }_{\mathrm{CK}}$

\begin{tabular}{|c|c|c|c|c|c|c|}
\hline \multirow[b]{2}{*}{ Lags } & \multicolumn{2}{|c|}{$\operatorname{VAR}(\mathbf{1})$} & \multicolumn{2}{|c|}{ VAR(2) } & \multicolumn{2}{|c|}{$\operatorname{VAR}(3)$} \\
\hline & LM-Stat & Prob & LM-Stat & Prob & LM-Stat & Prob \\
\hline 1 & 218,341 & o,ooo & 131,771 & o,ooo & 99,855 & 0,003 \\
\hline \multirow[t]{2}{*}{2} & 127,671 & 0,000 & 114,417 & 0,000 & 88,390 & 0,023 \\
\hline & \multicolumn{2}{|c|}{$\operatorname{VAR}(4)$} & \multicolumn{2}{|c|}{ VAR(5) } & \multicolumn{2}{|c|}{ VAR(6) } \\
\hline Lags & LM-Stat & Prob & LM-Stat & Prob & LM-Stat & Prob \\
\hline $\mathbf{1}$ & 124,250 & 0,000 & 87,002 & 0,030 & 62,728 & 0,522 \\
\hline 2 & 89,449 & 0,020 & 83,137 & 0,054 & 66,152 & 0,403 \\
\hline
\end{tabular}

Fonte: Elaboração própria.

Gráfico A1 - Teste de estabilidade dos parâmetros para o VAR(6) com cred cK $_{1}$

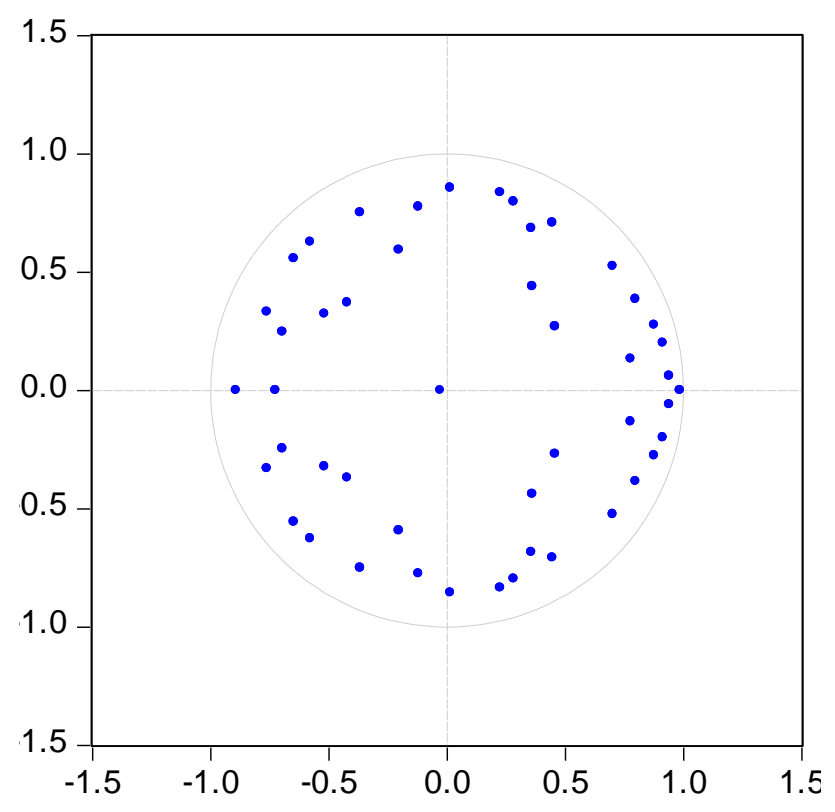

Fonte: Elaboração própria. 
Tabela A2 - Testes LM de autocorrelação residual para o VAR $\operatorname{com} \operatorname{cred}_{M}$

\begin{tabular}{|c|c|c|c|c|c|c|}
\hline \multirow[b]{2}{*}{ Lags } & \multicolumn{2}{|c|}{$\operatorname{VAR}(\mathbf{1})$} & \multicolumn{2}{|c|}{$\operatorname{VAR}(2)$} & \multicolumn{2}{|c|}{ VAR(3) } \\
\hline & LM-Stat & Prob & LM-Stat & Prob & LM-Stat & Prob \\
\hline $\mathbf{1}$ & 178,112 & 0,000 & 137,207 & 0,000 & 97,948 & 0,004 \\
\hline \multirow[t]{2}{*}{2} & 124,694 & 0,000 & 110,573 & 0,000 & 88,791 & 0,022 \\
\hline & \multicolumn{2}{|c|}{$\operatorname{VAR}(4)$} & \multicolumn{2}{|c|}{ VAR(5) } & \multicolumn{2}{|c|}{ VAR(6) } \\
\hline Lags & LM-Stat & Prob & LM-Stat & Prob & LM-Stat & Prob \\
\hline $\mathbf{1}$ & 127,175 & 0,000 & 86,436 & 0,032 & 63,263 & 0,503 \\
\hline 2 & 87,493 & 0,027 & 79,415 & 0,093 & 59,584 & 0,633 \\
\hline
\end{tabular}

Fonte: Elaboração própria.

Gráfico Az - Teste de estabilidade dos parâmetros para o VAR(6) $\operatorname{com}_{\text {cred }}$

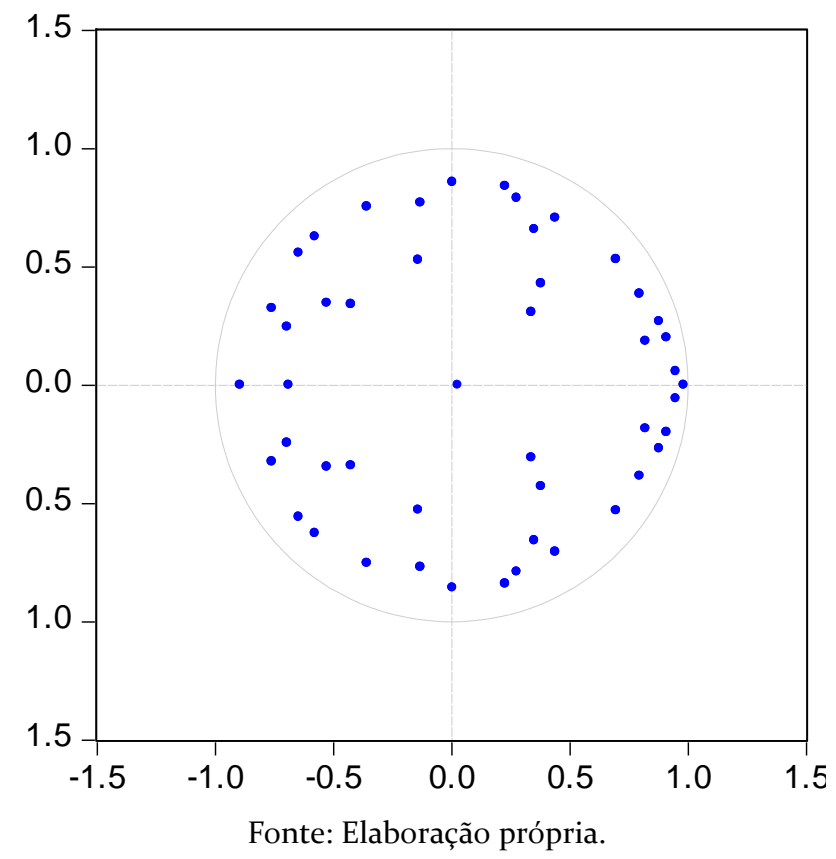


Tabela $A_{3}$ - Variáveis instrumentais

\begin{tabular}{|c|c|}
\hline Tabelas 3 e 4 & Tabelas 4 e 5 \\
\hline$E(\mathrm{p})_{t-2}$ & $E(\mathrm{p})_{t-2}$ \\
\hline$d(e)_{t-2}$ & $d(e)_{t-2}$ \\
\hline $\mathrm{i}_{\mathrm{t}-2}$ & $\mathrm{i}_{\mathrm{t}-2}$ \\
\hline $\mathrm{d}(\mathrm{ic})_{\mathrm{t}-2}$ & $\mathrm{~d}\left(\mathrm{icfmi}_{\mathrm{t}-2}\right.$ \\
\hline$E(\mathrm{p})_{t-3}$ & $E(\mathrm{p})_{t-3}$ \\
\hline$d(e)_{t-3}$ & $d(e)_{t-3}$ \\
\hline $\mathrm{i}_{\mathrm{t}-3}$ & $\mathrm{i}_{\mathrm{t}-3}$ \\
\hline $\mathrm{d}(\mathrm{ic})_{\mathrm{t}-}$ & $\mathrm{d}(\mathrm{icfmi})_{\mathrm{t}-3}$ \\
\hline$E(\mathrm{p})_{t-4}$ & $E(\mathrm{p})_{t-4}$ \\
\hline$d(e)_{t-4}$ & $d(e)_{t-4}$ \\
\hline $\mathrm{i}_{\mathrm{t}-4}$ & $\mathrm{i}_{\mathrm{t}-4}$ \\
\hline $\mathrm{d}(\mathrm{ic})_{\mathrm{t}-4}$ & $\mathrm{~d}(\mathrm{icfmi})_{\mathrm{t}-4}$ \\
\hline
\end{tabular}

Fonte: Elaboração própria. 